\title{
Histological Changes in Proximal and Distal Convoluted Tubules of Kidney of Albino Rats after Exposure to Mosquito Coil Smoke Inhalation
}

\section{Nazia Siddique, ${ }^{1}$ Masooma Ahmed, ${ }^{2}$ Maria Ilyas, ${ }^{3}$ Rukhsana Jabeen, ${ }^{4}$ Abdul Hannan Jawad, Zafar Iqbal $^{6}$}

\begin{abstract}
Objective: To evaluate the effects of the mosquito coil smoke (MCS) inhalation on histology of proximal (PCT) and distal (DCT) convoluted tubules of kidney in Wistar Albino rats.

Methods: This study was approved by the Ethical Committee of PGMI, Lahore. 24 Wistar rats were selected and randomly divided into 3 groups, each containing eight animals. Group A was control; Group B and C were experimental groups and were exposed to mosquito coil smoke inhalation for 8 hours/day for two and four weeks respectively. Kidney tissue of albino rats was dissected, examined and analyzed histologically.

Results: The results of MCS inhalation in histological sections of group B and C showed marked cellular necrosis and vacuolization in PCT (proximal convoluted tubule) of the kidney as compared to the group A. protein cast was absent in PCT of all groups. DCT (distal convoluted tubules) in group B and C showed marked necrosis, vacuolization and protein cast. Necrosis was more marked in group $\mathrm{C}$ treated with mosquito coil smoke for 4 weeks.

Conclusion: The results indicate that pyrethroids in mosquito coil smoke though considered least toxic pesticides, are very harmful. Exposure of pyrethroids can induce adverse changes in tubules of kidney.

Key Words: MCS Mosquito Coil Smoke, Pyrethroids, PCT Proximal convoluted tubules, DCT Distal convoluted tubules.

How to Cite: Siddique N, Ahmed M, Ilyas M, Jabeen R, Jawad A.H, Iqbal Z. histological changes in proximal and distal convoluted tubules of kidney of albino rats after exposure to mosquito coil smoke inhalation. Esculapio.2020;16(04):87-91.
\end{abstract}

DOI: https://doi.org/10.51273/esc20.2516419

\section{Introduction}

$\mathrm{M}$ osquitoes are the main agent which can cause various vector-borne diseases. Most common diseases in Pakistan are Malaria and Dengue Fever. During the second decade of $21^{\text {st }}$ century the Pakistan encountered the dengue epidemics. As no vaccine is available against dengue fever the only way of protec-

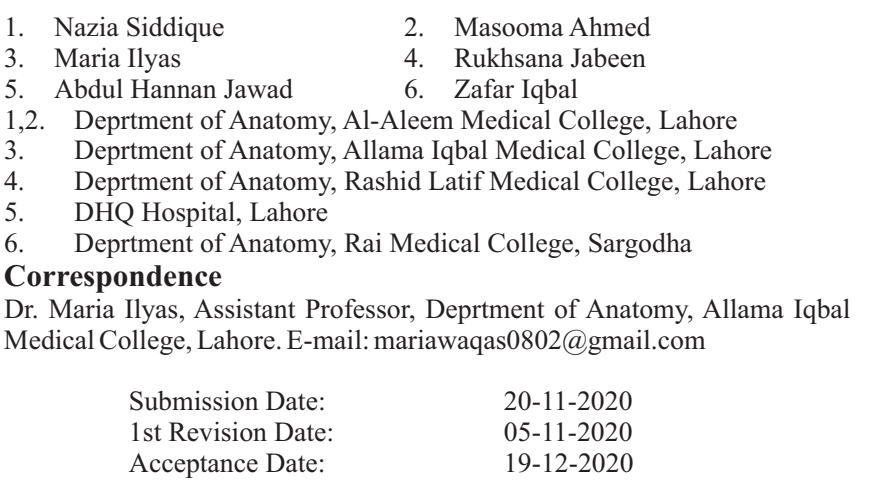

tion is prevention. People tend to wear long sleeved clothes with long trousers to prevent mosquito bites, sleep under bed nets, use topical repellent and burn repellent coils. As mosquito coils are cheap and easily available so it is most commonly used method to kill mosquitos. ${ }^{1}$ Previous studies suggested that mosquito coils containing pyretheroid as an active ingredient can help in controlling mosquito borne diseases. ${ }^{2}$ Mosquito coils smoke contain pyrethrin, dtransallethrin, aldehyde etc. Traditional coils are made from pyrethrum extracted from chrysanthemum cinnerariae folium plant. Pyrethrin is a natural pyreth-rum and pyretheroids are synthetic derivatives of pyrethrins. ${ }^{3}$

One of previous study estimated that smoke produced by burning one mosquito coil was equivalent to smoke produced by burning approximately 75-137 cigarettes. ${ }^{4}$ It was discovered that in some developing 
countries the use of unauthorized mosquito coils may induce human poisoning. ${ }^{5}$ Pyretheroid poisioning produced a defect in cardiac conduction. ${ }^{6}$ Vacuolar degeneration, necrosis, thrombosis and vasculitis in heart were also reported. Sore throat, nausea, vomiting and abdominal pain are acute effects of Pyretheroid ingestion. ${ }^{7}$

Pyretheroid based mosquito coils induce neurotoxicity by oxidative damage in brain. ${ }^{8}$ According to previous studies the action on voltage gated sodium channel and receptor gated ion channels are main cause of neurotoxicity. ${ }^{9}$ The decrease in motor activity was produced by Pyrethroid. ${ }^{6}$ Prolonged and uninterrupted exposure to mosquito coil smoke containing allethrin induces toxic changes in males testis including destruction of tubular archi-tecture, epithelial cell disruption, increase in the size of the lumen and necrotic spermatozoa. ${ }^{10}$ In female, Pyrethroid may disrupt the estrous cycle, endocrine glands may have showed increase in weight especially thyroids and adrenals." Pyrethrin and pyrethroids enter the body by many routes including ingestion with food, inhalation via coil smoke or burning or directly in contact with the skin. These chemicals are metabolized very rapidly in human body. Nervous system is the main primary target of the pyrethroid toxicity. ${ }^{8}$ Chronic exposure of pyrethroides produce many toxic effects including skin allergy, asthma, irritation of nose, throat and damages heart, lungs, liver and kidneys. ${ }^{8,9}$ Pyrethroids (DTA) are prone to induce toxicity at cellular levels leading to necrosis and tissue damage. Neurotoxic effects of chemicals emitted via mosquito coil smoke are extensively reported. ${ }^{10}$ Many of the studies conducted in the past have established morphological, biochemical and cellular toxicity in the gastrointestinal and especially in the inhalants respiratory system. ${ }^{11}$ Prolonged coil smoke exposure lead to adverse behavioral and neurological side effects in rat's offspring during prenatal and early postnatal period. ${ }^{12}$ It decreased sperm concentration and induced liver toxicity. ${ }^{7}$ This study was designed to observe the toxic effects mosquito coil smoke on histology of kidney.

\section{Methods}

The animal study was conducted in the animal house of Anatomy department, PGMI, Lahore. The study protocol and procedures were approved by the
Ethical Committee of PGMI, Lahore.

This was an experimental study, randomized control trial. Albino rats of Wister strain were obtained from animal house, PGMI, Lahore. Inclusion criteria were healthy animal of mix gender with average weight of 180-200 grams. Animals were weighed and examined thoroughly for any gross morbidity. They were individually kept in climate-controlled conditions of temperature $23 \pm 0.5^{\circ} \mathrm{C}$ and 12 hours light / dark cycles in an undisturbed well ventilated room. They were provided with standard rat food and water ad libitum. Rats were acclimatization in their cages for 15 days.

After acclimatization, the animals were randomly divided using Stat Trek random number generator into three groups A, B and C. Each group contained 8 rats. Group A was not exposed to mosquito coil and served as control. Group B and C were experimental group and were exposed to mosquito coil smoke. During the experiment rats were kept in their designated cages and allowed to inhale the MCS as whole body inhalation. The mosquito coil was burnt and then placed in the center of room. The animal cages were positioned equidistant from the mosquito coil so that all rats inhale equal amount of MCS. Group A was control and was not exposed to any mosquito coil smoke. Group B and group C were experimental and were exposed to mosquito coil smoke for 8 hours/ day for two and four weeks respectively. All animals were sacrificed under deep anesthesia 24 hours after exposure to their last dose. Kidneys (right and left) were dissected out. Weight of both kidneys was recorded carefully on electronic weighing scale. All the animals were euthanized by decapitation. Their bodies were disposed-off by burying in burial ground at PGMI, Lahore. Kidneys were washed with normal saline and fixed in $10 \mathrm{ml}$ of neutral $10 \%$ buffered formalin. Tissue processing was done by using automatic tissue processor and then embedded in paraffin. 3-4 $\mu \mathrm{m}$ thick serial paraffin sections were obtained and stained with standard haematoxylin and eosin (H\&E) reagent for the histopathological examination. Slides were examined under light microscope (ACCU-SCOPE 3000-LED Microscope) at 10X and 40X magnifications. Proximal and distal convoluted tubules were obser-ved in 5 fields of view in each kidney. The cells of proximal and distal convoluted tubules were examined for the presence of cellular necrosis, cellular vacuolization and protein cast. The observations were recorded in MS Word ${ }^{\circledR}$ and 
Excel® data sheet. Data was analyzed using SPSS $21 \cdot 0$. Qualitative analysis of parameters was carried out by using Pearsons chi square test or/and fisher exact test, at $5 \%$ level of significance. A p-value $\leq$ 0.05 was considered as statistically significant.

\section{Results}

Proximal convoluted tubules were lined by simple cuboidal epithelium with prominent brush border, round nuclei with prominent nucleoli. The cell size and cell shape remain constant in PCT when three groups were compared. Necrosis of PCT was absent in group A while present in group B and C and was statistically significant when all the three groups were compared. (Table 1, Figure 1) Cytoplasmic vacuoles were observed in PCT cells in treated groups and statistically significant when compared with control (Figures 2,3,4). Protein cast was not significant statistically in PCT. Cellular necrosis, cytoplasmic vacuoles and luminal protein cast in PCT and DCT were absent in group A. Distal convoluted tubles were recognized by larger more clearly defined lumen, lined by simple cuboidal epithelium, closely packed nuclei and lack of brush border. The cell size and shape remain constant in all three groups. Group A showed no signs of cellular necrosis, vacuolization and protein cast. Cellular mecrosis, vaculization and protein cast were present in both group $\mathrm{B}$ and $\mathrm{C}$ and were statistically significant. (Table 2) (Figure 2,3,4)

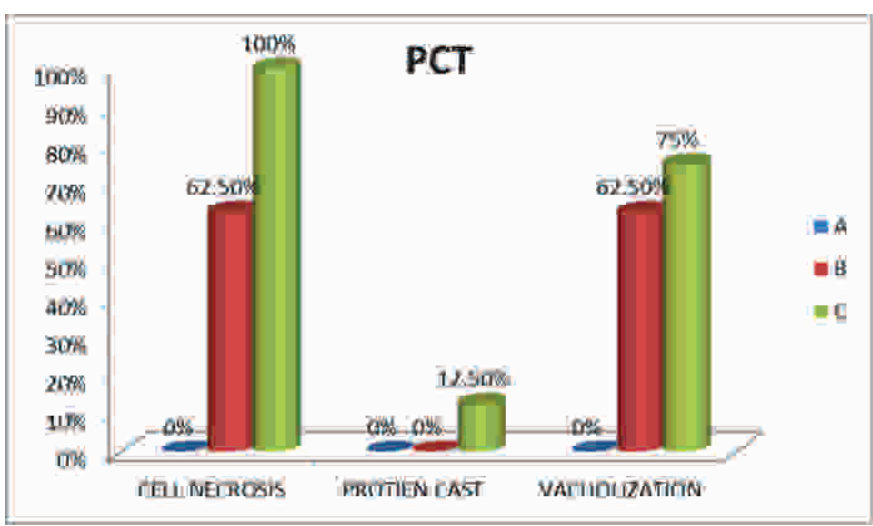

,Figure 1: Bar Chart Showing Comparison Percentage of Necrosis, Vacuolization and Protein Cast in PCT and DCT between Groups I, II, III.

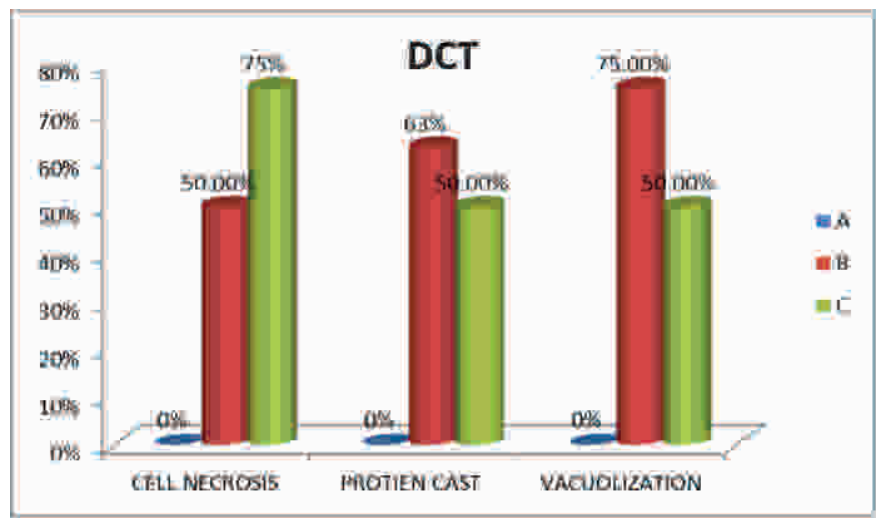

Figure 2. Photomicrograph of the Kidney from the Control group A Showing PCT Lined by Cuboidal Epithelium with Brush Border (Brown Arrow) and

Table 1: Showing Percentage of Cellular Necrosis, Protein cast and Vacuolization in PCT in Group A, B and C.

\begin{tabular}{|c|c|c|c|c|c|c|c|c|}
\hline \multirow[t]{2}{*}{ PCT } & \multirow[t]{2}{*}{ Status } & \multicolumn{2}{|c|}{ Group A } & \multicolumn{2}{|c|}{ Group B } & \multicolumn{2}{|c|}{ Group C } & \multirow{2}{*}{$\begin{array}{c}\chi^{2} \text {-test } \\
\text { (p-value) }\end{array}$} \\
\hline & & $\mathrm{n}$ & $\%$ & $\mathbf{N}$ & $\%$ & $\mathbf{N}$ & $\%$ & \\
\hline \multirow[t]{2}{*}{ Cell necrosis } & Normal & 8 & \multirow[t]{2}{*}{$0 \%$} & 3 & \multirow[t]{2}{*}{$62.5 \%$} & 0 & \multirow[t]{2}{*}{$100 \%$} & \multirow[t]{2}{*}{$0.000^{* * *}$} \\
\hline & Necrotic & 0 & & 5 & & 8 & & \\
\hline \multirow[t]{2}{*}{ Protein cast } & Absent & 8 & \multirow[t]{2}{*}{$0 \%$} & 8 & \multirow[t]{2}{*}{$0 \%$} & 7 & \multirow[t]{2}{*}{$12.5 \%$} & \multirow[t]{2}{*}{0.352} \\
\hline & Present & 0 & & 0 & & 1 & & \\
\hline \multirow[t]{2}{*}{ Vacuolization } & Normal & 8 & \multirow[t]{2}{*}{$0 \%$} & 3 & \multirow[t]{2}{*}{$62.5 \%$} & 2 & \multirow[t]{2}{*}{$75 \%$} & \multirow[t]{2}{*}{$0.006^{* *}$} \\
\hline & Vacuolized & 0 & & 5 & & 6 & & \\
\hline
\end{tabular}

${ }^{*} \mathrm{p}$-value $\leq 0.05$ is considered to be statistically significant.

Table 2: Showing Percentage of Cellular Necrosis, Protein Cast and Vacuolization in DCT in Group A, B and C

\begin{tabular}{ccccccccc}
\hline \multirow{2}{*}{ DCT } & Status & \multicolumn{2}{c}{ Group A } & \multicolumn{2}{c}{ Group B } & \multicolumn{2}{c}{ Group C } & $\begin{array}{c}\chi^{2} \text {-test } \\
\text { (p-value) }\end{array}$ \\
\cline { 2 - 7 } Cell necrosis & & $\mathbf{n}$ & $\mathbf{\%}$ & $\mathbf{n}$ & $\mathbf{\%}$ & $\mathbf{N}$ & $\mathbf{\%}$ & \\
& Normal & 8 & $0 \%$ & 4 & $50 \%$ & 2 & $75 \%$ & $0.008^{* *}$ \\
& necrotic & 0 & & 4 & & 6 & & \\
Protein cast & Absent & 8 & $0 \%$ & 3 & $62.5 \%$ & 4 & $50 \%$ & $0.024^{*}$ \\
& Present & 0 & & 5 & & 4 & & \\
Vacuolization & normal & 8 & $0 \%$ & 2 & $75 \%$ & 4 & $50 \%$ & NA \\
& Vacuolized & 0 & & 6 & & 4 & & \\
\hline
\end{tabular}

\footnotetext{
${ }^{*}$ p-value $\leqslant 0.05$ is considered to be statistically significant.
} 
DCT lined by cuboidal epithelium (black arrow) Renal corpusle (blue arrow) with central glomerulus (green arrow) surrounded by bowman's space (red arrow) and blood vessels are also present (yellow arrow). $H \& E$ stain $10 X$

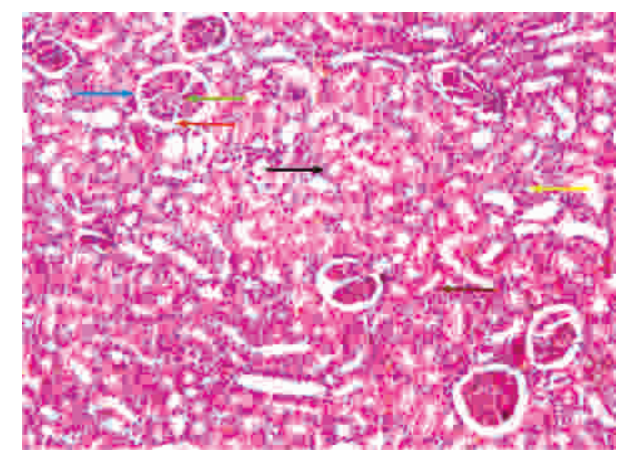

Figure 3. Photomicrograph of the kidney from the group B (right) and group C (left) showing necrosis of both PCT (yellow arrow) and DCT (blue arrow). Cytoplasmic vacuoles are present (green arrow). Congested glomerulus (black arrow) and interstitial hemorrhage (brown arrow) is seen. Protein casts are present in DCT (redarrow). H\&E stain . $10 \mathrm{X}$

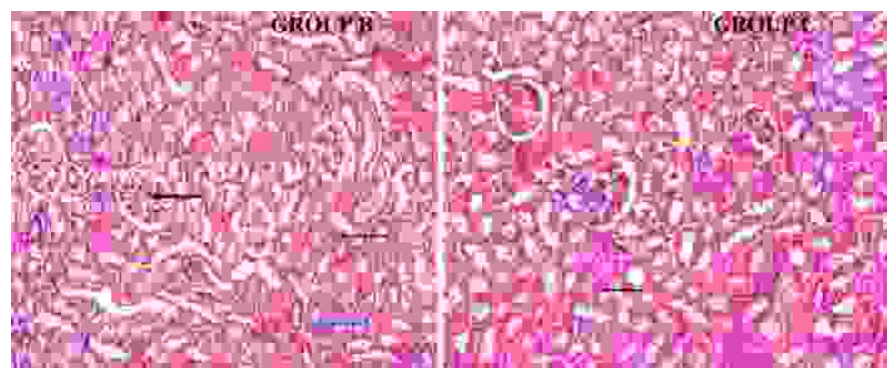

Figure 4: Photomicrograph of the kidney from the group B(right) and group C (left) showing necrosis of renal tubules (blue arrow) and interstitial hemorrhage (green arrow). Cytoplasmic vacuolization of tubular epithelium (black arrow) and protein casts in DCT (green arrow) are seen H\&E stain. $40 X$

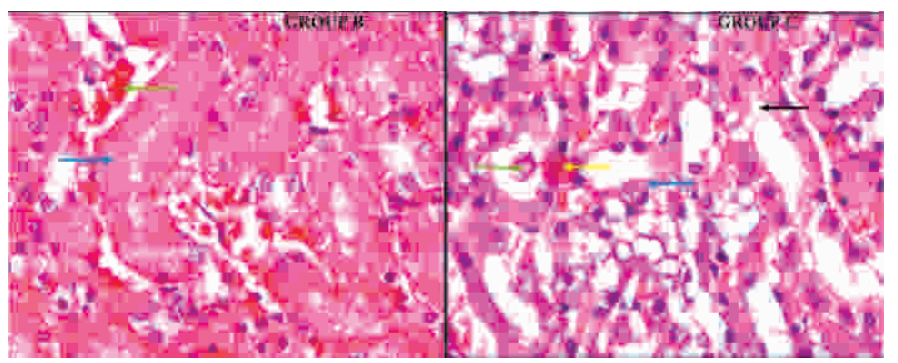

\section{Discussion}

The current study was designed to see the effects of mosquito coil smoke exposure on kidney tissue, using rat as a model. The experimental results demonstrated Pyrethroids (DTA) based mosquito coils are toxic in nature. Previously conducted studies have demons- trated that mosquito coil smoke inhalation shows signs of toxicity in liver and lung. ' many previously conducted studies proved adverse effects of prolonged inhalation mosquito coil smoke. Current study has focused on the histopathological effects on the hictoarchitecture of kidneys. ${ }^{5,8,13}$

The histological observations on kidney from treated groups $\mathrm{B}$ and $\mathrm{C}$ showed marked toxic changes. The kidney tubules of rat treated with mosquito coil smoke inhalation showed necrosis and vacuolization. This tubular necrosis and vacuolization was due to the accumulation of phospholipids, lipids and cholesterol deposits in the cell. ${ }^{13}$ Studies have shown that Pyrethroids act as potent inhi bitors of the mitochondrial complex I and oxygen, leading to their toxic effects on the mitochondria. The increased oxygen consumption causes an increase in the oxidative stress. It induces lipid peroxidation, protein oxidation and depletion of multiple antioxidant enzymes, which include glutathione, glutathione peroxidase, and catalase and superoxide dismutase activities 14. The increased oxygen consumption increases mitochondrial oxygen usage. Pyrethroids inhibit the ATP production by the cellular mitochondria. A principle molecular mode of adverse action of the synthetic pyrethroids is alteration of sodium potassium $(\mathrm{Na}+/$ $\mathrm{K}+$ ) pump kinetics. ${ }^{15}$ The sodium and water transport into cellular interior increases causing cellular fluid overload, disturbed protein synthesis and altered mechanical functions. These disturbed $\mathrm{Na}+/ \mathrm{K}+$ pump causes impairment of the oxidative phosphorylation that eventually results in hydrophilic degeneration, cellular swelling and vacuolization in the cytoplasm of renal tubular and parenchymal cells. ${ }^{14}$ Pyrethroids increase free radical formation which damages body cell. The formation of oxygen free radical is one of major factor in toxicity of pesticides. ${ }^{15}$ Voltage-gated sodium channels are mainly reported to be the primary target for toxicity of these coil smoke chemicals in humans. Research studies have depicted that all types of mammalian calcium channels are targets for allethrin at concentrations almost same as that reported for interaction with sodium channels. ${ }^{16}$ The micro molar concentration of both compounds inhibited glutamate and succinate sustained state 3 respirations in a concentration dependent manner. This inhibition is due to the effect of pyrethroids on mitochondrial transport system and also on the components of the respiratory chain. ${ }^{14}$

In our study we observed intraluminal cast formation in renal tubules. The matrix from the necrotic epithelial cell is the main cause for formation of this cast. ${ }^{17}$ This cast formation further obstructs the lumen of tubules. These findings suggest that inhalation of 
mosquito coil smoke cause nephrotoxicity. Their continuous usage causes harmful toxic effects in kidney. As these agents are easily absorbed in the body through inhalation.

\section{Conclusion}

The observations and results of present study clearly indicate that despite of being the least toxic pesticide, pyrethroids still have harmful and necrotizing effects, as exposure to pyrethroids cause nephrotoxicity. Present study will produce an awareness of the dangers of the excessive use of mosquito coils and restriction of unlimited use of pyrethroid insecticides especially at living places.

\section{Conflict of interest: None}

\section{References}

1. Agarwal AS, Yadav AK, Singh RA. Ameliorating effects of garlic oil against mosquito coil smoke induced histopathological changes in rat kidney. Int $\mathrm{J}$ Pharm Bio Sci. 2013;4(2):1112-6.

2. Msangi, S., Mwang'onde, B.J., Mahande, A.N. and Kweka, E.J., 2010. Field evaluation of the bioefficacy of three pyrethroid based coils against wild populations of anthropophilic mosquitoes in North Tanzania. Geographical medicine, 2(2): 116-120.

3. Zhang L, Jiang Z, Tong J, Wang Z, Han Z, Zhang J. Using charcoal as base material reduces mosquito coil emissions of toxins. Indoor air. $2010 \mathrm{Apr} ; 20(2)$ : 176-84.

4. Salvi D, Limaye S, Muralidharan V, Londhe J, Madas S, Juvekar S, Biswal S, Salvi S. Indoor particulate matter $<2.5 \mu \mathrm{m}$ in mean aerodynamic diameter and carbon monoxide levels during the burning of mosquito coils and their association with respiratory health. Chest. 2016 Feb 1;149(2):459-66.

5. Rahman MM. Insecticide substitutes for DDT to control mosquitoes may be causes of several diseases. Environmental Science and Pollution Research. 2013 Apr 1;20(4):2064-9.

6. Bhaskar, E.M., Moorthy, S., Ganeshwala, G. and Abraham, G., 2010. Cardiac conduction disturbance due to prallethrin (pyrethroid) poisoning. J Med Toxicol., 6(1): 27-30.

7. Taiwo, V.O., Nwagbara, N.D., Suleiman, R., Angbashim. and Zarma, M.J., 2008. Clinical signs and organ pathology in rats exposed to graded doses of pyrethroid containing mosquito coil smoke and aerosolized insecticidal sprays. African Journal of Biomedical Research, 11:97-104.

8. Vences-Mejía A, Gómez-Garduño J, Caballero-
Ortega H, Dorado-González V, Nosti-Palacios R, Labra-Ruíz N, Espinosa-Aguirre JJ. Effect of mosquito mats (pyrethroid-based) vapor inhalation on rat brain cytochrome P450s. Toxicology Mechanisms and Methods. 2012 Jan 1;22(1):41-6.

9. Neal, A.P., Yuan, Y. and Atchison, W.D., 2010. Allethrin differentially modulates voltage-gated calcium channel subtypes in rat PC12 cells. Toxicol Sci., 116: 604-613.

10. Madhubabu, G. and Yenugu, S., 2012. Effect of continuous inhalation of allethrin-based mosquito coil smoke in the male reproductive tract of rats. Inhal Toxicol., 24(3): 143-52.

11. Sangha, G.K., Kaur, K., Khera, K.S. and Singh, B., 2011. Toxicological effects of cypermethrin on female albino rats. Toxicol Int., 18(1): 5-8.

12. Sinha, C., Agrawal, A.K., Islam, F., Seth, K., Chaturvedi, R.K., Shukla, S. and Seth, P.K., 2004. Mosquito repellent (pyrethroid-based) induced dysfunction of blood-brain barrier permeability in developing brain. Int J Dev Neurosci., 22(1): 31-7.

13. Anusha C, Sankar R, Varunkumar K, Sivasindhuja G, Ravikumar V. Fourier transform-infrared spectroscopy as a diagnostic tool for mosquito coil smoke inhalation toxicity in Swiss Albino mice. Journal of Molecular Structure. 2017 Dec 5;1149:128-35.

14. Sahar, A., Abou, E.M., Sabik, L.M.E. and Shoukry, A., 2011. Pyretheroid toxic effects on some hormonal profile and biochemical markers among workers in pyretheroid insecticides company. Life Science Journal, 8(1):311-322.

15. Somia, I., Maghraby, E., Hamdy, A., Taha and Nabila, S. and Hassan., 2010. Effect of anthum graveolens L. extract on biochemical and histopathological alteration of deltamethrin in rats. J of Bioanal \& Biomed., 2(1): 008-012.

16. Soderlund DM. Molecular mechanisms of pyrethroid insecticide neurotoxicity: recent advances. Archives of toxicology. $2012 \mathrm{Feb} 1 ; 86(2): 165-81$.

17. Palm, F. and Nordquist, L., 2011. Renal tubulointerstial hypoxia: Cause and consequence of kidney dysfunction. Clinical and Experimental Pharmacology, 38(7): 474-480.

\section{Author's Contributions}

SN: Principal investigator, data collection, study design \& concept

AM: Literature review, data collection and interpretation of analysis.

IM: Data collection, critical evaluation, final editing of manuscript

JR: Data interpretation \& tables

JHA: Data collection \& statistical analysis

IZ: Manuscript writing 